\section{Acurácia da gordura corporal e do perímetro da cintura para predizer alterações metabólicas de risco cardiovascular em adolescentes}

\author{
Accuracy of body fat and waist circumference in predicting metabolic \\ abnormalities indicating cardiovascular risk in adolescents
}

Milena Miranda de Moraes' ${ }^{\prime}$, Gloria Valeria da Veiga'

\section{RESUMO}

Objetivo: Avaliar a acurácia do percentual de gordura corporal (\%GC) e do perímetro da cintura (PC) na predição de alterações metabólicas em adolescentes. Sujeitos e métodos: Estudo transversal com amostra probabilística de 573 estudantes de 12 a 19 anos da rede estadual de ensino de Niterói, RJ. Utilizou-se a Receiver Operating Characteristic Curve para avaliar a acurácia do \%GC e PC na predição de alterações de pressão arterial, perfil lipídico e glicemia. Resultados: Os melhores pontos de corte do \%GC foram $21 \%$ para meninos e $23 \%$ para as meninas, com sensibilidade (SE) e especificidade (ESP) de aproximadamente $60 \%$. Para o PC, $71 \mathrm{~cm}$ para meninos e $66 \mathrm{~cm}$ para meninas apresentaram melhor SE (55\% a 70\%) e ESP (55\% a 78\%). Conclusão: Mesmo os melhores pontos de corte identificam incorretamente proporções elevadas de adolescentes com alterações metabólicas, sugerindo cautela no uso desses indicadores para triagem de risco cardiovascular nessa faixa etária. Arq Bras Endocrinol Metab. 2014;58(4):341-51

Descritores

Adolescentes; gordura corporal; perímetro da cintura; hiperlipidemias; hipertensão arterial

\begin{abstract}
Objective: To evaluate the performance of percent body fat (\%BF) and waist circumference (WC) in predicting metabolic abnormalities indicating cardiovascular risk in adolescents. Subjects and methods: Cross-sectional study with a probabilistic sample of 573 adolescents aged 12 to 19 years from state public schools in Niterói, RJ. The Receiver Operating Characteristic Curve was used to evaluate the accuracy of $\mathrm{WC}$ and \%BF in predicting alterations in blood pressure, lipid profile, and blood glucose. Results: The best \%BF cutoff points were $21 \%$ for boys, and $23 \%$ for girls, with sensitivity (SE) and specificity (SP) of approximately $60 \%$. The best WC cutoff points were $71 \mathrm{~cm}$ for boys, and $66 \mathrm{~cm}$ for girls (SE: $55 \%$ to $70 \%$; SP: $55 \%$ to $78 \%$ ). Conclusion: Even the best cutoff points misidentified high proportions of adolescents with metabolic abnormalities. Caution is recommended in the use of these indicators for cardiovascular risk screening in this age group. Arq Bras Endocrinol Metab. 2014;58(4):341-51
\end{abstract}

Keywords

Adolescents; body fat; waist circumference; hyperlipidemias; hypertension
${ }^{1}$ Instituto de Nutrição Josué de Castro, Universidade Federal do Rio de Janeiro (UFRJ), Rio de Janeiro, RJ, Brasil

\section{INTRODUÇÃO}

$\mathrm{N}$ o Brasil, 20,5\% dos adolescentes apresentam excesso de peso, prevalência que aumentou em seis vezes entre meninos e três vezes entre meninas nas últimas quatro décadas (1). Esse avanço é preocupante, pois alterações como hipertensão arterial sistêmica, dis- lipidemias e diabetes melito tipo 2 já foram observadas em adolescentes obesos brasileiros (2) e aumentam o risco para doenças cardiovasculares na vida adulta (3).

$O$ índice de massa corporal (IMC) tem sido utilizado na avaliação de excesso de peso e obesidade em adolescentes por ser prático, de baixo custo e apresentar 
bom desempenho na identificação do excesso de gordura corporal (4). Todavia, esse índice não apresentou bom desempenho na identificação de adolescentes brasileiros com alterações metabólicas de risco cardiovascular (5). Em adolescentes israelenses obesos, essas alterações se correlacionaram mais fortemente à gordura corporal do que ao IMC (6).

Adolescentes com percentual de gordura corporal (\%GC) igual ou acima de $25 \%$ para meninos e $30 \%$ para meninas apresentaram maior risco para concentrações séricas elevadas de lipoproteínas não $\mathrm{HDL}$ e pressão arterial aumentada (7). O perímetro da cintura como indicador de acúmulo de gordura na região abdominal também apresentou associação positiva com pressão arterial sistólica e triglicerídeos séricos em adolescentes brasileiros (8). No entanto, os pontos de corte com melhor acurácia desses indicadores para identificação de morbidade nessa faixa etária ainda não foram bem estabelecidos. O objetivo deste estudo foi avaliar o desempenho do perímetro da cintura e do \%GC, estimado pela bioimpedância elétrica na predição de alterações metabólicas de risco cardiovascular em adolescentes e identificar os melhores pontos de corte para tal predição.

\section{SUJEITOS E MÉTODOS}

Os dados são provenientes de estudo transversal que investigou a presença de fatores de risco para doenças cardiovasculares em adolescentes, desenvolvido com estudantes de 12 a 19 anos, do $6^{\circ}$ ano do ensino fundamental ao $3^{\circ}$ ano do ensino médio, da rede estadual de ensino de Niterói, RJ, no ano de 2003.

A amostra foi calculada com base na prevalência de hipercolesterolemia, estimada em $25 \%$ para adolescentes (9), por ser uma alteração de risco para doença cardiovascular muito prevalente nessa faixa etária. Considerando-se um intervalo de confiança de $95 \%$, precisão absoluta de 5 pontos percentuais, o efeito do desenho da amostra por conglomerado em um estágio (sorteio de turmas) e a perda de até $30 \%$, conforme experiência obtida no estudo-piloto, a amostra inicial foi estimada em 780 adolescentes (4).

Foram sorteadas 28 turmas (com média de 28 alunos cada), distribuídas em 13 das 33 escolas que compunham a rede estadual de ensino da cidade de Niterói em 2002. Foram considerados inelegíveis adolescentes que apresentavam deficiência física que impossibilitasse a avaliação antropométrica, gestantes e adolescentes com obesidade secundária a outras doenças (informação obtida por entrevista com o adolescente).
A coleta de dados antropométricos foi feita por equipe de 11 avaliadores treinados e padronizados em medidas antropométricas, dentro da margem de erro aceitável pelo critério de Habicht (10), com sessões de retreinamento periódicas.

Os adolescentes foram pesados em balança eletrônica portátil modelo PPS (Kratos-Cas Balanças Eletrônicas, Embu, Brasil), com capacidade de até $150 \mathrm{~kg}$ e variação de $50 \mathrm{~g}$, descalços e trajando roupas leves. A estatura foi medida com estadiômetro portátil Leicester (United Kingdom), com extensão de $200 \mathrm{~cm}$ e variação de $0,1 \mathrm{~cm}$ com os adolescentes descalços, de calcanhares juntos e encostados no antropômetro e com a cabeça no plano horizontal de Frankfürt (11). A medida da estatura foi feita em duplicata, admitindo-se variação máxima de $0,5 \mathrm{~cm}$ entre as duas medidas e calculando-se a média. Com os dados de peso e estatura, calculou-se o IMC (peso/estatura ${ }^{2}$ ), e o status de peso dos adolescentes foi classificado, segundo critério da Organização Mundial de Saúde (12), considerando-se com excesso de peso aqueles com IMC, para sexo e idade, acima de 1 escore $\mathrm{Z}$, e com obesidade aqueles com IMC acima de 2 escores $\mathrm{Z}$.

$\mathrm{O}$ perímetro da cintura foi medido em duplicata, com fita métrica inextensível de $150 \mathrm{~cm}$ e variação de $0,1 \mathrm{~cm}$, no menor perímetro do tronco (13), admitindo-se variação máxima de $1 \mathrm{~cm}$ entre as duas medidas e obtendo-se a média.

$\mathrm{O}$ \%GC foi estimado por meio da bioimpedância elétrica, sistema perna-a-perna (TANITA ${ }^{\circledR}$ TBF-305). A densidade corporal foi obtida por equação fornecida pelo fabricante, específica para faixa etária e sexo, utilizando-se os valores de impedância (considerando a resistência), peso e estatura do adolescente, conforme descrito a seguir: sexo masculino: $\mathrm{DC}=0,872341$ $-\left[0,116448 \times \mathrm{P} \times\left(\mathrm{Z} / \mathrm{E}^{2}\right)\right]+\left[3,015403 \times\left(\mathrm{Z} / \mathrm{E}^{2}\right)\right]$ $+(0,001520 \times \mathrm{E})$; sexo feminino: $\mathrm{DC}=0,914826-$ $\left[0,097504 \times \mathrm{P} \times\left(\mathrm{Z} / \mathrm{E}^{2}\right)\right]+\left[2,085173 \times\left(\mathrm{Z} / \mathrm{E}^{2}\right)\right]+$ $(0,001171 \times \mathrm{E})$, em que: $\mathrm{DC}=$ densidade corporal; $\mathrm{P}=$ peso $(\mathrm{kg}) ; \mathrm{Z}=$ impedância $; \mathrm{E}=$ estatura $(\mathrm{cm})$. Para cálculo do \%GC, a partir da densidade corporal, foi utilizada equação específica para a faixa etária em questão (14). Para estimativa de prevalências de excesso de gordura corporal, utilizaram-se valores de $\%$ GC iguais ou acima de $25 \%$ para meninos e $30 \%$ para meninas (7).

A pressão arterial foi medida com manômetro digital (Pro Check $\left.{ }^{\circledR}\right)(8)$, sendo considerada a média de duas aferições, realizadas com intervalo de dois minutos, com 
diferença máxima permitida de $5 \mathrm{mmHg}$ entre as duas medidas. Os adolescentes estavam sentados, usando roupas sem mangas, e permaneceram em repouso por, pelo menos, cinco minutos antes das medições (15).

A coleta de sangue foi realizada por laboratorista devidamente treinado, nos adolescentes em jejum de 12 horas, mediante agendamento prévio. Para garantir o jejum, antes da coleta os adolescentes eram questionados se haviam ingerido qualquer alimento, incluindo balas e doces, nas últimas 12 horas. Foram coletados, aproximadamente, $10 \mathrm{~mL}$ de sangue em 3 tubos tipo Vacutainer, que foram armazenados em isopor com gelo seco e transportados, imediatamente após a coleta, ao laboratório de Fisiologia da Nutrição e Desenvolvimento da Universidade Estadual do Rio de Janeiro, onde o material foi centrifugado e analisado. As dosagens de colesterol total, HDL-colesterol (hight density lipoprotein), triglicerídeos e glicose plasmática foram feitas pelo método enzimático automatizado $E x$ terss Plus. O LDL-colesterol (low density lipoprotein) foi calculado usando a fórmula de Friedwald: LDL- colesterol $=$ colesterol total $-($ HDL-colesterol + triglicerídeos/5) (16).

Os pontos de corte utilizados para caracterizar alterações metabólicas foram: colesterol total $\geq 150 \mathrm{mg} /$ $\mathrm{dL}$, LDL-C $\geq 100 \mathrm{mg} / \mathrm{dL}$, triglicerídeos $\geq 100 \mathrm{mg} / \mathrm{dL}$ e HDL-colesterol < $45 \mathrm{mg} / \mathrm{dL}$ (17); glicemia de jejum $\geq$ $100 \mathrm{mg} / \mathrm{dL}$ (18). Considerou-se pressão arterial elevada quando: $\mathrm{PAS} / \mathrm{PAD} \geq 120 / 80 \mathrm{mmHg}$ ou $\geq$ percentil 90 (p90) segundo sexo, idade e estatura para idade para os menores de 18 anos e PAS/PAD $\geq 130 / 85 \mathrm{mmHg}$ para os maiores de 18 anos (15). Além da identificação da presença ou ausência de cada uma dessas alterações, criou-se a variável risco para doença cardiovascular (RDCV) que foi categorizada em quatro níveis: nenhuma alteração, 1 alteração, 2 alterações e 3 ou mais alterações.

Nas análises estatísticas, levaram-se em consideração o efeito do desenho de amostra por conglomerado em um estágio (sorteio de turmas) e a expansão da amostra pelo peso relativo, utilizando o módulo para amostras complexas Complex Samples do programa SPSS versão 19.0. Utilizou-se o procedimento general linear model para comparação das médias e o teste Qui-quadrado para comparação das frequências das variáveis entre adolescentes com e sem excesso de gordura corporal e adolescentes com valores de perímetro da cintura abaixo e igual ou maior que o percentil 90 (p90) da distribuição da população avaliada. Em todas as análises, aceitou-se o valor de $\mathrm{p}<0,05$ para significância estatística.
Para avaliar a acurácia do \%GC e do perímetro da cintura na predição de cada uma das alterações metabólicas, de pressão arterial e da variável RDCV, utilizou-se a Receiver Operator Characteristic curve (curva ROC). Determinaram-se a área sob a curva (ASC) e seus respectivos intervalos de confiança (IC) de $95 \%$, os quais não deveriam incluir o valor 0,50 para indicar desempenho significativo das medidas na predição dos desfechos investigados (19). Ao interpretar os resultados, considerou-se que quanto mais próxima de 1,0 fosse a ASC, melhor a acurácia do \% GC e do perímetro da cintura na predição das alterações investigadas. Utilizando a curva ROC, identificaram-se também os pontos de corte desses indicadores que apresentaram melhor sensibilidade (SE) e especificidade (ESP) para predição de cada uma das alterações.

Em função da influência da idade na variação da composição corporal, nas análises efetuadas, os valores de \%GC e perímetro da cintura foram ajustados por idade pelo método dos resíduos, conforme descrito por Willett e cols. (20). Todas as análises foram estratificadas por sexo.

$\mathrm{O}$ estudo de base que gerou os dados analisados neste trabalho foi aprovado pelo Comitê de Ética em Pesquisa do Hospital Universitário Clementino Fraga Filho, da Universidade Federal do Rio de Janeiro, e só participaram do estudo os adolescentes que quiseram e que tiveram o termo de consentimento esclarecido assinado pelos pais, quando menores de 18 anos.

\section{RESULTADOS}

Dos 757 alunos elegíveis, obtiveram-se dados antropométricos, bioquímicos e de pressão arterial para 573 adolescentes (resposta de $75,7 \%$ ), sendo $68,3 \%$ do sexo feminino, que foram incluídos na análise do poder de predição do perímetro da cintura com esses indicadores. $\mathrm{Na}$ avaliação da acurácia do \% GC, foram utilizados os dados de 567 estudantes. A prevalência de excesso de peso observada foi de $17,6 \%$ (20,4\% entre os meninos e $16,3 \%$ entre meninas, $\mathrm{p}=0,315)$ e $3,4 \%$ dos adolescentes eram obesos $(2,7 \%$ entre meninos e $3,7 \%$ em meninas, $\mathrm{p}=0,615)$.

Colesterol total elevado e baixas concentrações de HDL-colesterol foram as alterações metabólicas mais frequentes, observadas em $56 \%$ da população de estudo, seguidas por valores elevados de LDL colesterol $(41 \%)$, glicose $(22 \%)$ e triglicerídeos $(20 \%)$, sem diferença significativa entre sexos. A pressão arterial eleva- 
da foi observada em $25 \%$ dos adolescentes, sendo mais prevalente nos meninos do que nas meninas ( $52 \%$ vs. $15 \%, \mathrm{p}<0,01)$. Quase todos os adolescentes apresentaram, ao menos, uma das alterações de risco cardiovascular investigadas, independente de sexo $(92,5 \%$ das meninas e $95,2 \%$ dos meninos), de status de gordura corporal (99,3\% naqueles com excesso e $91,7 \%$ naqueles sem excesso de gordura corporal) e de localização na curva de valores de perímetro da cintura $(98,3 \%$ naqueles com perímetro da cintura $\geq$ p90 e $92,9 \%$ naqueles com perímetro da cintura $<$ p90) (dados não exibidos).

As médias de peso, IMC, perímetro da cintura e pressão arterial sistólica foram maiores, e a média de es- tatura foi menor nos adolescentes com excesso de gordura corporal quando comparados aos sem excesso em ambos os sexos. Maiores médias $(\mathrm{p}<0,05)$ de colesterol, LDL e triglicerídeos, assim como maiores frequências de alterações metabólicas, também foram observadas nas meninas com excesso de gordura corporal (Tabela 1).

Os adolescentes com perímetro da cintura maior ou igual ao p90 da distribuição da própria população de estudo tiveram as maiores médias de peso, IMC, gordura corporal, pressão arterial e lipídios séricos (com exceção do HDL-colesterol) quando comparados àqueles com valores abaixo do p90. A frequência de adolescentes com alterações de risco cardiovascular, de modo geral, também foi maior naqueles com perímetro da cintura

Tabela 1. Média expandida (erro-padrão) das variáveis antropométricas, de composição corporal e bioquímicas e prevalência expandida (erro-padrão) de alterações metabólicas em adolescentes com e sem excesso de gordura corporal, segundo sexo. Estudantes de escolas públicas, Niterói, RJ, 2003

\begin{tabular}{|c|c|c|c|c|}
\hline \multirow[b]{2}{*}{ Variável } & \multicolumn{2}{|c|}{ Sexo masculino } & \multicolumn{2}{|c|}{ Sexo feminino } \\
\hline & $\begin{array}{c}\text { Com excesso de GC } \\
(\mathrm{N}=43)\end{array}$ & $\begin{array}{c}\text { Sem excesso de GC } \\
(N=162)\end{array}$ & $\begin{array}{l}\text { Com excesso de GC } \\
(\mathrm{N}=78)\end{array}$ & $\begin{array}{c}\text { Sem excesso de GC } \\
(N=284)\end{array}$ \\
\hline \multicolumn{5}{|c|}{ Média (EP)`- } \\
\hline Idade (anos) & $17,2(0,4)$ & $16,9(0,3)$ & $16,9(0,4)$ & $16,6(0,3)$ \\
\hline Peso (kg) & $74,2(2,0)^{\mathrm{a}}$ & $62,2(1,0)$ & $63,9(1,6)^{\mathrm{a}}$ & $52,4(0,5)$ \\
\hline Estatura (cm) & $172,2(0,9)^{b}$ & $174,8(0,8)$ & $158,5(0,7)^{b}$ & $162,1(0,8)$ \\
\hline IMC (kg/m²) & $24,8(0,5)^{\mathrm{a}}$ & $20,3(0,2)$ & $25,3(0,5)^{\mathrm{a}}$ & $19,9(0,1)$ \\
\hline$\% G C$ & $28,7(0,3)^{\mathrm{a}}$ & $18,3(0,4)$ & $36,8(0,9)^{\mathrm{a}}$ & $20,2(0,4)$ \\
\hline $\mathrm{PC}(\mathrm{cm})$ & $79,8(1,5)^{\mathrm{a}}$ & $70,8(0,5)$ & $75,3(1,2)^{\mathrm{a}}$ & $65,4(0,3)$ \\
\hline PA sistólica (mmHg) & $127,5(2,2)^{b}$ & $121,0(1,8)$ & $113,2(2,2)^{\mathrm{a}}$ & $106,6(1,0)$ \\
\hline PA diastólica (mmHg) & $72,0(1,9)$ & $70,0(1,3)$ & $68,0(1,3)^{b}$ & $64,3(0,7)$ \\
\hline Colesterol (mg/dL) & $156,9(4,9)$ & $148,9(4,4)$ & $177,1(6,7)^{b}$ & $155,8(3,4)$ \\
\hline LDL-C (mg/dL) & $91,3(4,7)$ & $86,2(3,3)$ & $112,6(4,9)^{\mathrm{a}}$ & $94,4(3,3)$ \\
\hline $\mathrm{HDL}-\mathrm{C}(\mathrm{mg} / \mathrm{dL})$ & $45,0(1,6)$ & $47,0(1,8)$ & $46,2(2,2)$ & $46,6(1,6)$ \\
\hline Triglicerídeos (mg/dL) & $95,0(6,7)$ & $81,6(2,9)$ & $92,4(5,8)^{b}$ & $76,1(3,2)$ \\
\hline Glicose (mg/dL) & $88,9(2,2)$ & $91,6(1,9)$ & $88,4(1,9)$ & $90,4(1,7)$ \\
\hline \multicolumn{5}{|c|}{ Prevalência (EP) ${ }^{\ddagger}$} \\
\hline PA elevada & $59,9(9,2)$ & $50,2(5,6)$ & $22,8(6,2)$ & $12,5(3,1)$ \\
\hline $\mathrm{CT} \geq 150 \mathrm{mg} / \mathrm{dL}$ & $65,9(7,3)$ & $48,0(6,0)$ & $82,7(3,9)^{\mathrm{a}}$ & $51,6(5,0)$ \\
\hline $\mathrm{LDL}-\mathrm{C} \geq 100 \mathrm{mg} / \mathrm{dL}$ & $40,1(7,6)$ & $34,7(5,3)$ & $66,4(7,7)^{\mathrm{a}}$ & $37,3(5,0)$ \\
\hline $\mathrm{HDL}-\mathrm{C}<45 \mathrm{mg} / \mathrm{dL}$ & $63,4(6,7)$ & $52,6(7,8)$ & $58,6(8,7)$ & $55,9(7,8)$ \\
\hline $\mathrm{TG}>100 \mathrm{mg} / \mathrm{dL}$ & $41,6(9,1)^{\mathrm{b}}$ & $20,2(3,5)$ & $31,8(7,4)^{b}$ & $14,8(2,7)$ \\
\hline Glicose $\geq 100 \mathrm{mg} / \mathrm{dL}$ & $20,9(7,8)$ & $30,6(7,2)$ & $14,9(4,3)$ & $20,4(6,1)$ \\
\hline \multicolumn{5}{|c|}{ Alterações de risco } \\
\hline Nenhuma & $1,2(1,2)$ & $5,9(2,4)$ & $0,5(0,5)^{\mathrm{a}}$ & $9,3(3,2)$ \\
\hline Uma & $14,2(6,2)$ & $22,3(3,5)$ & $12,3(3,4)^{\mathrm{a}}$ & $35,3(3,4)$ \\
\hline Duas & $22,8(6,4)$ & $26,7(4,9)$ & $14,2(3,9)$ & $20,9(2,7)$ \\
\hline Três ou mais & $61,8(8,6)$ & $45,1(4,6)$ & $73,0(4,9)^{\mathrm{a}}$ & $34,5(4,7)$ \\
\hline
\end{tabular}

${ }^{\star} \geq 25 \%$ para meninos e $\geq 30 \%$ para meninas; † Comparação de médias por general linear model; ${ }^{\ddagger}$ Comparação de proporções por teste Qui-quadrado; ${ }^{\text {a }} \mathrm{p} \leq 0,01 ;{ }^{\mathrm{b}} \mathrm{p}<0,05$. GC: gordura corporal; IMC: índice de massa corporal; PA: pressão arterial; CT: colesterol total; LDL-C: low-density lipoprotein cholesterol; HDL-C: high-density lipoprotein cholesterol; TG: triglicerídeos séricos. 
superior ao p90 da população, mas significância estatística só foi observada para os triglicerídeos nos meninos, pressão arterial nas meninas e colesterol total para ambos os sexos (Tabela 2).

A acurácia do \%GC e do perímetro da cintura na predição de alterações de risco cardiovascular está apresentada na tabela 3 . O perímetro da cintura teve melhor capacidade preditiva para identificar alterações do perfil lipídico e de pressão arterial em adolescentes do sexo masculino, enquanto o \% GC apresentou melhor capacidade preditiva em adolescentes do sexo feminino.
Os melhores pontos de corte de \%GC na predição de alterações de risco foram de, aproximadamente, $20 \%$ para meninos, com sensibilidade e especificidade de $60 \%$, e variaram entre $22 \%$ e $25 \%$ para meninas, com sensibilidade variando de $59 \%$ a $65 \%$ e especificidade de 58 a $65 \%$. Para o perímetro da cintura, os pontos de corte variaram entre $69,8 \mathrm{~cm}$ e $72,6 \mathrm{~cm}$ para meninos, com sensibilidade de $60 \%$ a $67 \%$ e especificidade de $43 \%$ a $78 \%$, e entre $66,3 \mathrm{~cm}$ e $67,7 \mathrm{~cm}$ para meninas, com sensibilidade de $55 \%$ a $65 \%$ e especificidade de $55 \%$ a $64 \%$ (Figuras 1 e 2 ).

Tabela 2. Média expandida (erro-padrão) das variáveis antropométricas, de composição corporal e bioquímicas e prevalência expandida (erro-padrão) de alterações metabólicas em adolescentes acima e abaixo do percentil $90^{*}$ do perímetro da cintura, segundo sexo. Estudantes de escolas públicas Niterói, RJ, 2003

\begin{tabular}{|c|c|c|c|c|}
\hline \multirow[b]{2}{*}{ Variável } & \multicolumn{2}{|c|}{ Sexo masculino } & \multicolumn{2}{|c|}{ Sexo feminino } \\
\hline & $\begin{array}{c}P C \geq p 90 \\
(N=22)\end{array}$ & $\begin{array}{l}\mathrm{PC}<\mathrm{p} 90 \\
(\mathrm{~N}=185)\end{array}$ & $\begin{array}{c}P C \geq p 90 \\
(N=36)\end{array}$ & $\begin{array}{l}P C<p 90 \\
(N=330)\end{array}$ \\
\hline \multicolumn{5}{|c|}{ Média (EP)`- } \\
\hline Idade (anos) & $17,1(3,4)$ & $17,0(0,3)$ & $16,9(0,5)$ & $16,6(0,3)$ \\
\hline Peso (kg) & $88,2(3,2)^{a}$ & $62,2(0,9)$ & $74,9(2,4)^{\mathrm{a}}$ & $53,0(0,5)$ \\
\hline Estatura (cm) & $179,8(1,3)^{\mathrm{a}}$ & $173,6(0,8)$ & $162,0(0,9)$ & $161,3(0,7)$ \\
\hline IMC (kg/m²) & $27,2(0,7)^{\mathrm{a}}$ & $20,6(0,3)$ & $28,5(0,8)^{\mathrm{a}}$ & $20,4(0,1)$ \\
\hline GC (\%) & $28,4(0,8)^{\mathrm{a}}$ & $19,6(0,6)$ & $40,7(1,9)^{\mathrm{a}}$ & $22,0(0,6)$ \\
\hline $\mathrm{PC}(\mathrm{cm})$ & $87,4(1,6)^{\mathrm{a}}$ & $71,2(0,4)$ & $83,6(1,7)^{\mathrm{a}}$ & $66,1(0,3)$ \\
\hline PA sistólica (mmHg) & $131,2(3,2)^{b}$ & $121,4(1,6)$ & $117,5(2,3)^{\mathrm{a}}$ & $107,1(0,9)$ \\
\hline PA diastólica (mmHg) & $72,9(3,3)$ & $70,2(1,1)$ & $68,9(1,7)^{b}$ & $64,7(0,7)$ \\
\hline Colesterol (mg/dL) & $164,1(7,7)^{\mathrm{b}}$ & $149,0(4,1)$ & $172,5(6,4)^{b}$ & $158,9(3,5)$ \\
\hline LDL-C (mg/dL) & $95,7(8,0)^{\mathrm{a}}$ & $86,3(3,0)$ & $107,2(5,7)$ & $97,1(3,3)$ \\
\hline $\mathrm{HDL}-\mathrm{C}(\mathrm{mg} / \mathrm{dL})$ & $46,6(2,3)$ & $46,5(1,7)$ & $46,8(2,5)$ & $46,5(1,5)$ \\
\hline Triglicerídeos (mg/dL) & $109,1(8,3)^{b}$ & $82,0(2,5)$ & $92,5(7,3)$ & $77,8(3,1)$ \\
\hline Glicose (mg/dL) & $87,1(3,0)$ & $91,5(1,8)$ & $89,0(2,5)$ & $90,2(1,6)$ \\
\hline \multicolumn{5}{|c|}{ Prevalência (EP) ${ }^{\ddagger}$} \\
\hline PA elevada & $72,6(10,6)$ & $49,6(5,2)$ & $36,3(9,4)^{b}$ & $12,7(2,8)$ \\
\hline $\mathrm{CT} \geq 150 \mathrm{mg} / \mathrm{dL}$ & $73,3(9,8)^{b}$ & $49,5(5,4)$ & $78,6(8,1)^{\mathrm{b}}$ & $56,0(5,0)$ \\
\hline LDL-C $\geq 100 \mathrm{mg} / \mathrm{dL}$ & $38,6(11,5)$ & $35,5(4,8)$ & $53,9(10,7)$ & $42,4(5,1)$ \\
\hline $\mathrm{HDL}-\mathrm{C}<45 \mathrm{mg} / \mathrm{dL}$ & $55,5(12,6)$ & $55,4(7,2)$ & $61,2(11,7)$ & $55,9(7,4)$ \\
\hline $\mathrm{TG}>100 \mathrm{mg} / \mathrm{dL}$ & $56,8(10,1)^{\mathrm{a}}$ & $21,6(3,1)$ & $36,0(12,0)$ & $16,3(2,9)$ \\
\hline Glicose $\geq 100 \mathrm{mg} / \mathrm{dL}$ & $12,2(7,6)$ & $30,6(6,6)$ & $16,8(9,2)$ & $19,8(5,5)$ \\
\hline \multicolumn{5}{|c|}{ Alterações de risco } \\
\hline Nenhuma & 0,0 & $5,3(2,0)$ & $2,6(1,9)$ & $8,0(2,7)$ \\
\hline Uma & $9,9(5,8)$ & $21,5(3,2)$ & $8,6(4,9)^{b}$ & $32,8(3,4)$ \\
\hline Duas & $26,3(10,8)$ & $26,2(4,4)$ & $17,5(4,9)$ & $20,0(2,5)$ \\
\hline Três ou mais & $63,7(12,2)$ & $47,0(4,2)$ & $71,3(5,8)^{\mathrm{a}}$ & $39,2(4,6)$ \\
\hline
\end{tabular}

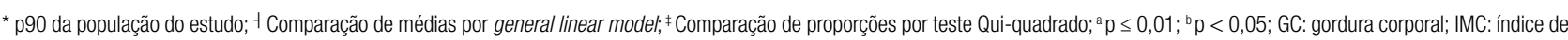
massa corporal; PA: pressão arterial; CT: colesterol total; LDL-C: low-density lipoprotein cholesterol; HDL-C: high-density lipoprotein cholesterol; TG: triglicerídeos séricos. 
Tabela 3. Área sob a curva ROC (ASC) de percentual de gordura e perímetro da cintura ajustados por idade na predição de alterações metabólicas em adolescentes. Estudantes de escolas públicas, Niterói, RJ, 2003

\begin{tabular}{|c|c|c|c|c|c|c|}
\hline \multirow{3}{*}{ Variável } & \multicolumn{6}{|c|}{ Sexo masculino } \\
\hline & \multicolumn{3}{|c|}{$\% G C(N=205)$} & \multicolumn{3}{|c|}{$\mathrm{PC}(\mathrm{N}=207)$} \\
\hline & ASC & IC 95\% & $p$ & ASC & IC 95\% & $p$ \\
\hline Colesterol total & 0,61 & $0,53-0,69$ & 0,007 & 0,63 & $0,55-0,70$ & 0,002 \\
\hline LDL-colesterol & 0,54 & $0,45-0,62$ & 0,396 & 0,56 & $0,48-0,64$ & 0,164 \\
\hline HDL-colesterol & 0,50 & $0,42-0,58$ & 0,984 & 0,52 & $0,44-0,60$ & 0,713 \\
\hline Triglicerídeos & 0,65 & $0,56-0,74$ & 0,002 & 0,64 & $0,54-0,74$ & 0,003 \\
\hline Glicose & 0,45 & $0,36-0,54$ & 0,241 & 0,44 & $0,35-0,52$ & 0,177 \\
\hline Pressão arterial & 0,54 & $0,46-0,62$ & 0,325 & 0,61 & $0,54-0,69$ & 0,004 \\
\hline \multicolumn{7}{|c|}{ Alterações metabólicas } \\
\hline Três ou mais & 0,58 & $0,50-0,66$ & 0,048 & 0,60 & $0,52-0,68$ & 0,014 \\
\hline \multirow[t]{2}{*}{ Pelo menos uma } & 0,62 & $0,46-0,79$ & 0,208 & 0,71 & 0,53-0,90 & 0,031 \\
\hline & \multicolumn{6}{|c|}{ Sexo feminino } \\
\hline \multirow[t]{2}{*}{ Variável } & \multicolumn{3}{|c|}{$\% G C(N=362)$} & \multicolumn{3}{|c|}{$\mathrm{PC}(\mathrm{N}=366)$} \\
\hline & ASC & IC 95\% & p-valor & ASC & IC 95\% & p-valor \\
\hline Colesterol total & 0,67 & $0,61-0,72$ & $<0,001$ & 0,57 & $0,51-0,63$ & 0,027 \\
\hline LDL-colesterol & 0,63 & $0,57-0,69$ & $<0,001$ & 0,56 & $0,50-0,62$ & 0,052 \\
\hline HDL-colesterol & 0,45 & $0,39-0,51$ & 0,108 & 0,48 & $0,42-0,54$ & 0,517 \\
\hline Triglicerídeos & 0,56 & $0,48-0,64$ & 0,117 & 0,57 & $0,49-0,65$ & 0,068 \\
\hline Glicose & 0,51 & $0,43-0,59$ & 0,788 & 0,49 & $0,41-0,57$ & 0,841 \\
\hline Pressão arterial & 0,71 & $0,63-0,78$ & $<0,001$ & 0,70 & $0,62-0,78$ & $<0,001$ \\
\hline \multicolumn{7}{|c|}{ Alterações metabólicas } \\
\hline Três ou mais & 0,65 & $0,59-0,71$ & $<0,007$ & 0,61 & $0,55-0,67$ & 0,001 \\
\hline Pelo menos uma & 0,67 & $0,58-0,76$ & 0,003 & 0,59 & $0,49-0,69$ & 0,131 \\
\hline
\end{tabular}

IC95\%: intervalo de confiança de 95; \%GC: percentual de gordura corporal; PC: perímetro da cintura; ASC: área sob a curva ROC.

\section{DISCUSSÃO}

O presente estudo é pioneiro no Brasil na identificação de pontos de corte de \%GC e perímetro da cintura para predição de alterações de pressão arterial, perfil lipídico e glicemia em uma amostra probabilística de adolescentes. Como resultado principal, destacamos que os pontos de corte dessas medidas, com melhor sensibilidade e especificidade para a identificação de adolescente com tais alterações, foram $20 \%$ para meninos e variaram entre $22 \%$ e $25 \%$ para meninas de percentual de gordura corporal, e aproximadamente $72 \mathrm{~cm}$ e $66 \mathrm{~cm}$ de perímetro de cintura para meninos e meninas, respectivamente. Esses valores são inferiores aos que têm sido propostos na literatura como indicadores de excesso de gordura corporal e de perímetro da cintura elevado, todavia, mesmo esses melhores pontos de corte não apresentaram boa acurácia na predição das alterações metabólicas, já que a sensibilidade e especificidade, de modo geral, não ultrapassaram $70 \%$.
Em relação ao \%GC, verificou-se que os pontos de corte de $25 \%$ para os meninos e $30 \%$ para meninas, já anteriormente utilizados para indicar excesso de gordura corporal em adolescentes $(7,21)$, apresentaram baixa sensibilidade e alta especificidade na identificação de concentrações séricas elevadas de colesterol total e triglicerídeos em adolescentes do sexo masculino e elevação de colesterol total, LDL colesterol e hipertensão arterial em adolescentes do sexo feminino. Mesmo para adolescentes com três ou mais alterações metabólicas, a sensibilidade desses pontos de corte, especialmente para as meninas, não ultrapassou $44 \%$. Esses resultados indicam que os pontos de corte de \%GC preconizados na literatura poderiam subestimar a presença de alterações metabólicas de risco cardiovascular, já que tais alterações já poderiam estar presentes em adolescentes antes de atingirem aqueles limites (falsos-negativos). Em outro estudo realizado no Brasil com crianças e adolescentes de 8 a 18 anos do sexo masculino (22), 

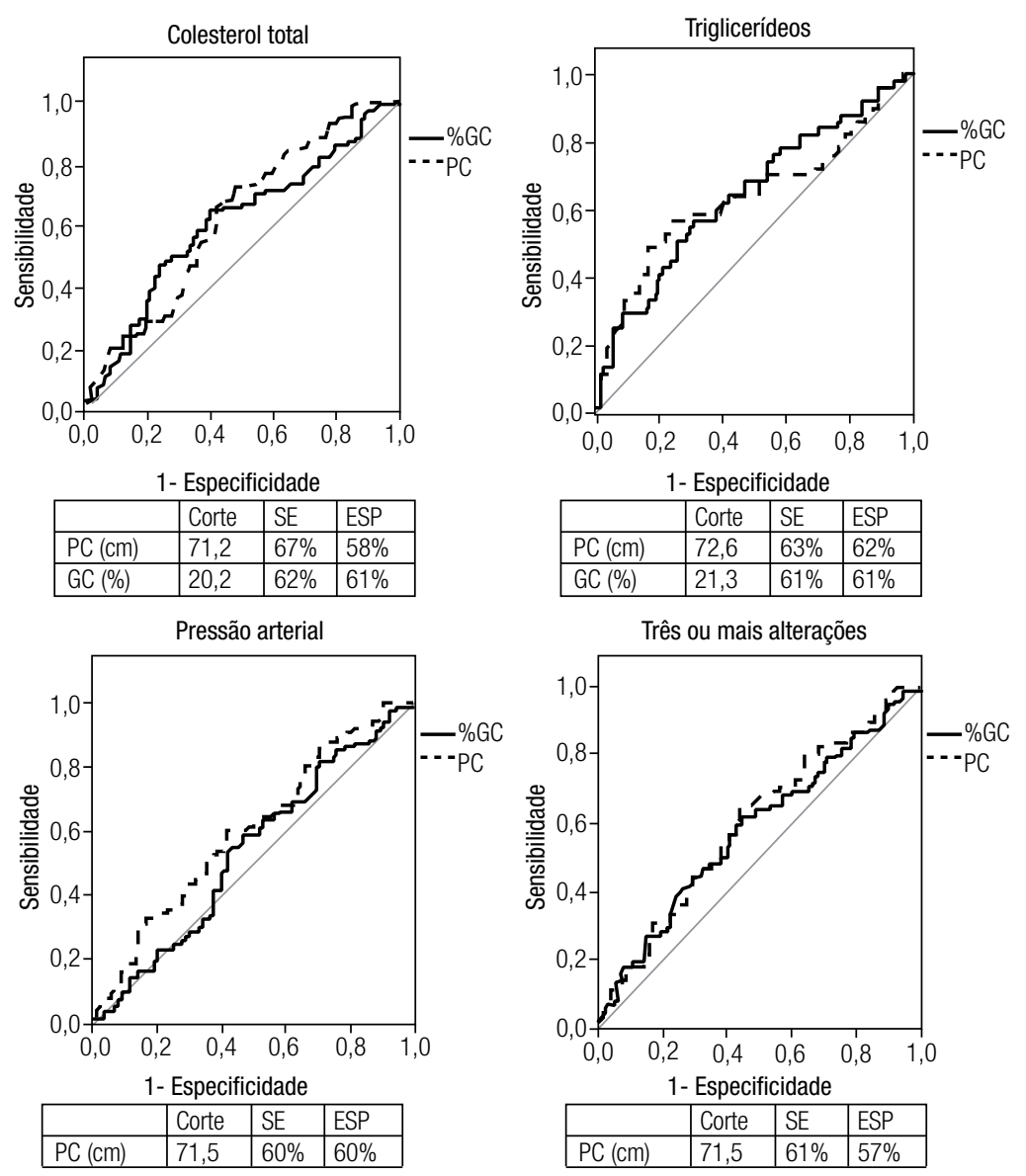

Figura 1. Curva ROC, melhores pontos de corte, sensibilidade (SE) e especificidade (ESP) do percentual de gordura corporal (\%GC) e do perímetro da cintura (PC) ajustados por idade na predição de alterações metabólicas em adolescentes do sexo masculino.

foram estabelecidos pontos de corte do \%GC obtido por DEXA (Dual-Energy X-Ray Absorptiometry), entre $15 \%$ e $25 \%$ para a predição de pressão arterial elevada, definida pelos mesmos critérios do nosso estudo, com grande variação na sensibilidade (31\% a $71 \%$ ) e especificidade $(65 \%$ a $100 \%)$, indicando que a capacidade preditiva do \%GC para alterações na pressão arterial ainda não é muito evidente nessa faixa etária, corroborando com nossos achados.

Em pesquisa realizada na Turquia (23), a acurácia do \%GC, estimado pela área adiposa do braço, para predição de hipertensão arterial sistêmica, foi semelhante à encontrada no nosso estudo para os meninos, mas nas meninas as ASC, que variaram de 0,58 a 0,55 , foram inferiores à observada no nosso estudo $(0,71)$, indicando pior acurácia. Os pontos de corte do \%GC com melhor sensibilidade e especificidade observados nos adolescentes turcos variaram entre $24,5 \%$ e $30,8 \%$ para meninos e entre $41,4 \%$ e $41,9 \%$ para as meninas, superiores aos observados no nosso estudo, principal- mente para as meninas $(25,2 \%)$. Tal diferença poderia ser atribuída ao maior percentual de gordura corporal observado nas adolescentes turcas, que variou de $33 \%$ a $37 \%$, enquanto no nosso estudo foi $23,5 \%$. Todavia, o uso de métodos distintos para estimativa do \%GC prejudica tal comparação. Além disso, a prevalência de hipertensão arterial sistêmica nas adolescentes turcas (entre 7\% e 10\%) foi menor do que a observada no nosso estudo (14\%). Em estudo realizado na Suécia (24), o \%GC estimado por pletismografia apresentou acurácia superior à observada nos adolescentes de Niterói na predição de alterações componentes da síndrome metabólica em adolescentes, com ASC de 0,65 e 0,87 na predição de triglicerídeos $\geq 150 \mathrm{mg} / \mathrm{dL}$ em meninas e meninos, respectivamente, e ASC de 0,65 para meninos e 0,55 para meninas na predição de HDL-colesterol < $40 \mathrm{mg} / \mathrm{dL}$. A pletismografia parece ser um método de melhor desempenho na análise da composição corporal em crianças e adolescentes do que a BIA (25), o que poderia explicar as diferenças entre os estudos. No en- 

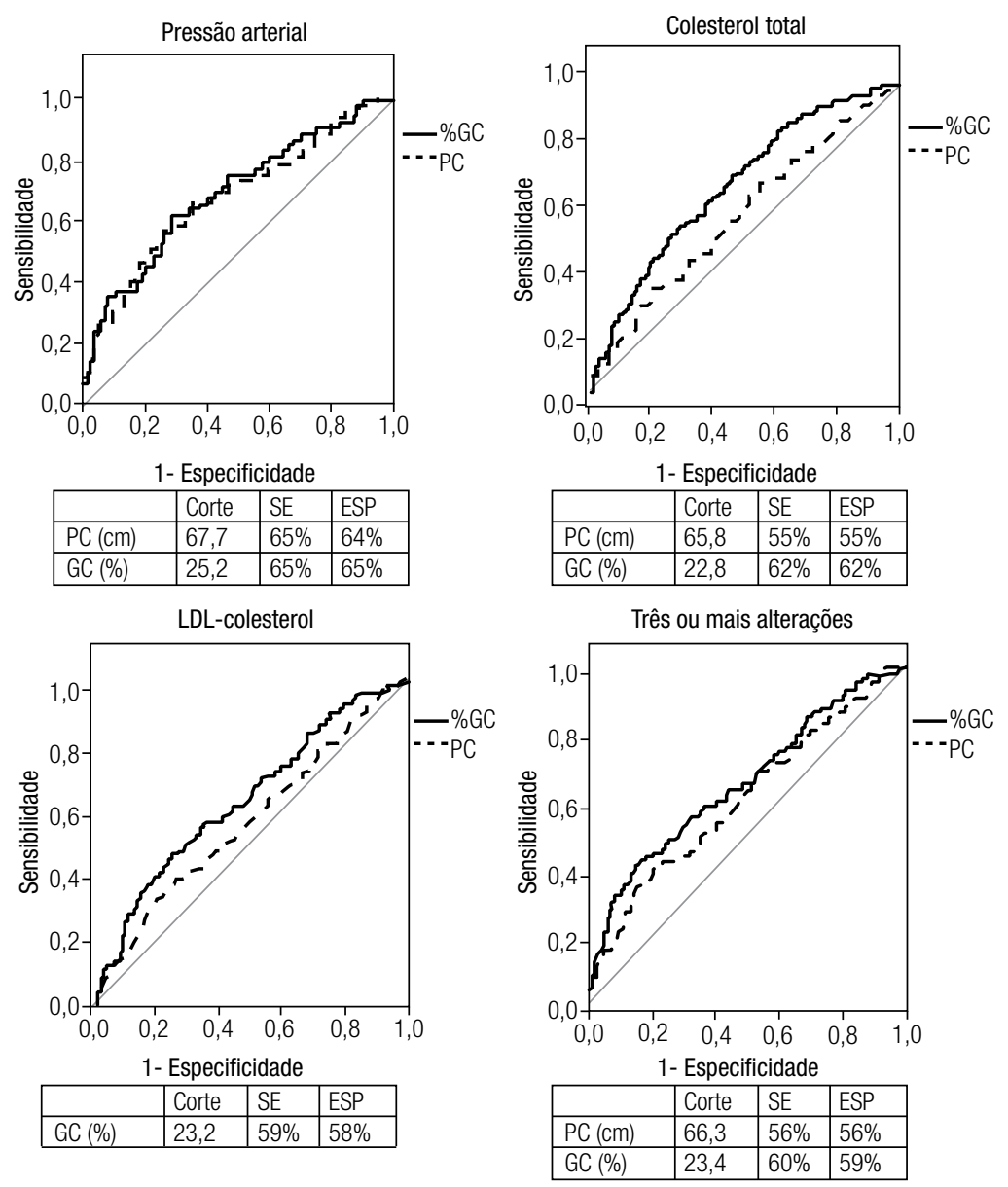

Figura 2. Curva ROC, melhores pontos de corte, sensibilidade (SE) e especificidade (ESP) do percentual de gordura corporal (\%GC) e do perímetro da cintura $(\mathrm{PC})$ ajustados por idade na predição de alterações metabólicas em adolescentes do sexo feminino.

tanto, sua aplicabilidade em estudos epidemiológicos é limitada, o que justifica a utilização de métodos mais práticos e viáveis, como a bioimpedância elétrica.

$\mathrm{Na}$ avaliação do desempenho do perímetro da cintura, a comparação dos nossos resultados com os de outros autores fica prejudicada devido a diferenças metodológicas na obtenção dessa medida, já que existem variadas definições para medidas feitas no mesmo local ou definições idênticas para medidas feitas em locais diferentes (26). Comparando a acurácia da medida no menor perímetro da cintura obtida no nosso estudo com a observada em adolescentes suecos (24), as ASC observadas nos meninos do nosso estudo para predição de alterações no perfil lipídico (variação de 0,63 a 0,64 ) foram inferiores às observadas na Suécia (variação de $0,65$ a 0,87$)$, mas semelhantes às observadas no sexo feminino $(0,58$ na Suécia e 0,57 no nosso estudo). Essas diferenças entre os meninos dos dois estudos, provavelmente, podem ser decorrentes do maior acúmulo de gordura na região abdominal nos adolescentes suecos, indicada pela maior média de perímetro da cintura em relação à nossa população, resultando em melhor acurácia dessa medida.

Valores entre $70 \mathrm{~cm}$ e $73 \mathrm{~cm}$ de perímetro da cintura apresentaram o melhor desempenho na identificação de adolescentes do sexo masculino com alterações de colesterol e triglicerídeos séricos, pressão arterial e três ou mais alterações de risco, com sensibilidade entre $58 \%$ e $78 \%$ e especificidade variando de $58 \%$ a $67 \%$. Entre as meninas, o desempenho do perímetro da cintura foi menos evidente, entretanto, valores de aproximadamente $66 \mathrm{~cm}$ identificaram alterações de colesterol total, pressão arterial e três ou mais alterações de risco. Tais valores, tanto para meninos quanto para meninas, equivalem ao percentil entre 50 e 55 da distribuição de perímetro da cintura da população investigada. Esse resultado se assemelha ao observado na detecção de três ou mais alterações de risco em crianças e adolescentes americanos 
(27), em que os melhores pontos de corte de perímetro da cintura para a faixa de 12 a 18 anos também corresponderam a cerca de p50 para meninos e meninas negros e brancos, embora os valores nesse percentil para os jovens americanos tenham sido um pouco mais elevados do que os observados no nosso estudo $(66 \mathrm{~cm}$ a $80 \mathrm{~cm}$ para meninos e $67 \mathrm{~cm}$ a $75 \mathrm{~cm}$ para as meninas).

No Brasil, o desempenho do perímetro da cintura em adolescentes tem sido mais investigado para predição de hipertensão arterial. Na cidade de Três de Maio, RS, foram observados valores com melhor desempenho entre $75,4 \mathrm{~cm}$ para meninos e $82,4 \mathrm{~cm}$ para meninas com SE de $78 \%$ e $100 \%$, respectivamente (28). Já em Londrina, PR, verificou-se que o ponto de corte no p 80 da distribuição de valores de perímetro da cintura da população investigada não apresentou boa capacidade preditiva (29), com acurácia semelhante à observada em nosso estudo para o sexo masculino e inferior para o sexo feminino.

$\mathrm{O}$ critério proposto pela International Diabetes $\mathrm{Fe}$ deration (IDF) (18) para diagnóstico da síndrome metabólica em adolescentes envolve, obrigatoriamente, o perímetro da cintura igual ou acima do p90, tendo como referência a população americana (30), associado a mais duas alterações (valores elevados de triglicerídeos, glicose e pressão arterial e baixos de HDL-colesterol). Os valores no p90 dessa referência entre 84,8 $\mathrm{cm}$ a $105,2 \mathrm{~cm}$ para meninos e de $82,7 \mathrm{~cm}$ a 101,1 $\mathrm{cm}$ para meninas de 12 a 18 anos chegam a superar os pontos de corte indicados para caracterizar obesidade abdominal em adultos $(90 \mathrm{~cm}$ para homens e $80 \mathrm{~cm}$ para mulheres) (31). Dessa forma, o critério da IDF propõe a utilização dos pontos indicados para adultos quando o p90 ultrapassa tais valores.

Tanto no nosso estudo quanto no estudo com adolescentes americanos (27), os valores de perímetro da cintura que foram capazes de predizer alterações de risco cardiovascular em adolescentes foram próximos à mediana das populações estudadas, o que indica quão baixa poderia ser a sensibilidade do p90 proposto pela IDF para identificar adolescentes em risco de síndrome metabólica ou mesmo de alterações isoladas nessa população. Ainda que o p90 da nossa população fosse utilizado na predição de risco, seria alta a proporção de adolescentes classificados como falso-negativos, visto que a utilização do valor de $81,6 \mathrm{~cm}$ (p90 para meninos) resultaria em sensibilidade de $12 \%$ a $24 \%$ para a predição de alterações de colesterol total, triglicerídeos, pressão arterial, e três ou mais alterações. O valor de $76,1 \mathrm{~cm}$ (p90 para as meninas) implicaria sensibilidade variando de $10 \%$ a $29 \%$ na identificação de alterações de colesterol total, pressão arterial e presença de três ou mais alterações. Em estudo realizado também na cidade de Niterói, RJ (32), a acurácia do p75 da distribuição de $\mathrm{PC}$ da população americana (30) (equivalente a valores de $74,3 \mathrm{~cm}$ a $87,0 \mathrm{~cm}$ para os meninos e de $82,7 \mathrm{~cm}$ a $98,0 \mathrm{~cm}$ para meninas), na predição de hipertensão arterial em adolescentes, resultou em $45 \%$ de sensibilidade, sugerindo que mesmo o p75 da distribuição da população americana como indicador de risco de hipertensão arterial pode ser muito elevado para adolescentes brasileiros.

Como discutido anteriormente, a baixa sensibilidade de um indicador, mesmo que resultando em melhor especificidade, pode repercutir no retardo de medidas preventivas para jovens em risco cardiovascular, o que não seria desejável no atual contexto de prevalência elevada de obesidade na adolescência. Cabe ressaltar que os melhores pontos de corte para \%GC e perímetro da cintura observados no nosso estudo não apresentaram melhor desempenho do que o IMC na predição de alterações metabólicas na mesma amostra de adolescentes, cujas ASC variaram entre 0,59 e 0,67 (5).

Apesar do desempenho insatisfatório dos dois indicadores de gordura corporal utilizados, neste estudo, o perímetro da cintura teve melhor poder de predição de alterações para os adolescentes do sexo masculino e $\mathrm{o} \% \mathrm{GC}$ apresentou melhor desempenho entre as meninas. Isso, provavelmente, se deve ao menor acúmulo de gordura abdominal e maior acúmulo de gordura corporal total nas meninas do que nos meninos (33).

Este estudo apresenta algumas limitações. O uso da bioimpedância elétrica para estimar o \%GC em adolescentes tem sofrido críticas, particularmente para estimativas individuais (34). No entanto, em estudos epidemiológicos, esse método tem apresentado boa acurácia na predição de gordura corporal nessa faixa etária (35) e acreditamos que o uso de uma equação específica para adolescentes tenha minimizado os erros de estimativa do percentual de gordura corporal. Outra limitação do estudo foi a impossibilidade de estimar pontos de corte específicos para todas as idades, ano a ano, devido ao tamanho da amostra, o que foi minimizado pelo ajuste das medidas de perímetro da cintura e \%GC pela idade.

Como o período da adolescência se caracteriza por mudanças corporais marcantes e estas ocorrem de maneira bastante peculiar a cada população e indivíduo, o estabelecimento de pontos de corte de medidas indicadoras de obesidade na predição de alterações metabólicas nessa faixa etária torna-se complexo. Além 
disso, a elevada prevalência de, pelo menos, uma dessas alterações, mesmo entre adolescentes sem excesso de gordura corporal total ou abdominal em nossa amostra, indica que a morbidade nessa faixa etária pode ser mais influenciada pelo potencial genético do que propriamente pela adiposidade, o que também dificulta a avaliação de relação entre medidas de adiposidade e risco de doenças. Inadequações no padrão dietético talvez também possam explicar as alterações no perfil bioquímico, independente da presença de obesidade. Elevado consumo de alimentos ricos em açúcar e gorduras foi observado em adolescentes brasileiros (36) e maior consumo de calorias e carboidratos complexos e simples se associou aos componentes da síndrome metabólica em adolescentes colombianos com excesso de peso (37). Todavia, a investigação do consumo alimentar não consiste em objetivo deste estudo.

Concluímos que as medidas de percentual de gordura corporal e perímetro da cintura não apresentaram bom desempenho na identificação de adolescentes com alterações de risco cardiovascular. Os melhores pontos de corte para identificar as alterações de risco nessa população foram inferiores aos propostos na literatura, $\mathrm{o}$ que indica que a utilização de referências de outras populações pode subestimar a ocorrência de morbidade associada ao excesso de adiposidade nos jovens brasileiros. Isso ressalta a importância de se elaborar referências nacionais para esse fim. Entretanto, mesmo os melhores pontos de corte encontrados a partir da distribuição de valores da população investigada predizem, incorretamente, a presença de alterações metabólicas em cerca de $40 \%$ dos adolescentes. Dessa forma, recomenda-se cautela na interpretação da acurácia, tanto do percentual de gordura corporal quanto do perímetro da cintura, na predição de risco cardiovascular nessa faixa etária.

Agradecimentos: ao Conselho Nacional de Desenvolvimento Científico e Tecnológico $(\mathrm{CNPq})$, pelo apoio financeiro, e à Secretaria de Ensino do Estado do Rio de Janeiro, por permitir a realização deste trabalho nas escolas participantes.

Declaração: os autores declaram não haver conflitos de interesse científico neste estudo.

\section{REFERÊNCIAS}

1. IBGE. Instituto Brasileiro de Geografia e Estatística - Pesquisa de Orçamentos Familiares 2008-2009: Antropometria e Estado Nutricional de Crianças, Adolescentes e Adultos no Brasil. Rio de Janeiro; 2010.

2. Pereira A, Guedes $A D$, Verreschi IT, Santos RD, Martinez TL. Obesity and its association with other cardiovascular risk factors in school children in Itapetininga, Brazil. Arq Bras Cardiol. 2009;93(3):253-60.
3. Kaikkonen JE, Jula A, Mikkilä V, Viikari JS, MoilanenT, NikkariT, et al. Childhood serum cholesterol ester fatty acids are associated with blood pressure 27 y later in the Cardiovascular Risk in Young Finns Study. Am J Clin Nutr. 2012;95(6):1422-31.

4. Vieira ACR, Alvarez MM, Marins VMR, Sichieri R, Veiga GV. Desempenho de pontos de corte do índice de massa corporal de diferentes referências na predição de gordura corporal em adolescentes. Cad Saúde Pública. 2006;22(8):1681-90.

5. Vieira ACR, Alvarez MM, Kanaan S, Sichieri R, Veiga GV. Índice de massa corporal para predizer hiperglicemia e alterações lipídicas em adolescentes brasileiros. Rev Saúde Pública. 2009;43(1):44-52.

6. Shalitin S, Phillip M. Frequency of cardiovascular risk factors in obese children and adolescents referred to a tertiary care center in Israel. Horm Res. 2008;69(3):152-9.

7. Williams DP, Going SB, Lohman TG, Harsha DW, Srinivasan SR, Webber LS, et al. Body fatness and risk for elevated blood pressure, total cholesterol, and serum lipoprotein ratios in children and adolescents. Am J Public Health. 1992;82(3):358-63.

8. Alvarez MM, Vieira AC, Sichieri R, Veiga GV. Associação das medidas antropométricas de localização de gordura central com os componentes da síndrome metabólica em uma amostra probabilística de adolescentes de escolas públicas. Arq Bras Endocrinol Metabol. 2008;52(4):649-57.

9. Gerber ZR, Zielinsky P. Fatores de risco de aterosclerose na infância: um estudo epidemiológico. Arq Bras Cardiol. 1997;69(4):231-6.

10. Habicht JP. Estandarizacion de métodos epidemiológicos cuantitativos sobre el terreno. Bol Oficina Sanit Panam. 1974;76:375-84.

11. Gordon CC, Chumlea WC, Roche AF. Stature, recumbent length, and weight. In: Lohman TG, Roche AF, Martorell R, editors. Anthropometric standardization reference manual. Champaign: $\mathrm{Hu}-$ man Kinetics Books; 1988. p. 3-8.

12. de Onis M, Onyango AW, Borghi E, Siyam A, Nishida C, Siekmann J. Development of a WHO growth reference for school-aged children and adolescents. Bull World Health Organ. 2007;85(9):660-7.

13. Callaway CW, Chumlea WC, Bouchard C, Himes JH, Lohman TG. Circumferences. In: Lohman TG, Roche AF, Martorell R, editors. Anthropometric Standardization Reference Manual. Champaign: Human Kinetics Book; 1988. p. 39-54.

14. Boileau RA. Body composition assessment in children and youth. In: Bar-Or O (Ed.). The child and adolescent athlete. Oxford: Blackwell Science; 1996. p. 523-37.

15. Sociedade Brasileira de Cardiologia/Sociedade Brasileira de $\mathrm{Hi}-$ pertensão/Sociedade Brasileira de Nefrologia. VI Diretrizes Brasileiras de Hipertensão. Arq Bras Cardiol. 2010;95(1 supl.1):1-51.

16. Friedwald WT, Levy RI, Fredrickson DS. Estimation of the concentration of lox-density lipoprotein in plasma without use of the preparative ultracentrifuge. Clin Chem. 1972;18(6):499-502.

17. Sociedade Brasileira de Cardiologia. I Diretriz de Prevenção da Aterosclerose na Infância e na Adolescência. Arq Bras Cardiol. 2005;85(supl.6):3-36.

18. International Diabetes Federation. The IDF consensus definition of metabolic syndrome in children and adolescents. Brussels, Belgium; 2007.

19. Zweig MH, Campbell G. Receiver-Operating Characteristic (ROC) Plots: a fundamental evaluation tool in clinical medicine. Clin Chem. 1993;39(4):561-77.

20. Willett WC, Howe GR, Kushi LH. Adjustment for total energy intake in epidemiologic studies. Am J Clin Nutr. 1997;65(suppl 4):1220S-8S.

21. Fernandes RA, Rosa CS, Silva CB, Bueno DR, Oliveira AR, Freitas Junior IF. Desempenho de diferentes valores críticos de índice de massa corporal na identificação de excesso de gordura corporal e obesidade abdominal em adolescentes. Rev Assoc Med Bras. 2007;53(6):515-9. 
22. Fernandes RA, Christofaro DGD, Buonani C, Araújo LR, Kassab YK, Cardoso JR, et al. The accuracy of national body fat cutoff levels in the prediction of elevated blood pressure among brazilian male adolescents. JTrop Pediatr 2010;56(3):208-9.

23. Mazicioglu MM, Yalcin BM, Ustunbas HB, Kurtoglu S. Anthropometric risk factors for elevated blood pressure in adolescents in Turkey aged 11-17. Pediatr Nephrol. 2010;25(10):2327-34.

24. Neovius M, Rossner SM, Vagstrand K, von Hausswolff-Juhlin YL, Hoffstedt J, Ekelund U. Adiposity measures as indicators of metabolic risk factors in adolescents. Obes Facts. 2009;2(5):294-301.

25. Azcona C, Koëk N, Frühbeck G. Fat mass by air displacement plethysmography and impedance in obese/non-obese children and adolescents. Int J Pediatr Obes. 2006;1(3):176-82.

26. Wang J, Thornton JC, Bari S, Williamson B, Gallagher D, Heymsfield $\mathrm{SB}$, et al. Comparisons of waist circumference measured at 4 sites. Am J Clin Nutr. 2003;77(2):379-84.

27. Katzmarzyk PT, Srinivasan SR, Chen W, Malina RM, Bouchard C, Berenson GS. Body mass index, waist circumference, and clustering of cardiovascular disease risk factors in a biracial sample of children and adolescents. Pediatrics. 2004;114(2):e198-205.

28. Beck CC, Lopes AS, Pitanga FJG. Indicadores antropométricos como preditores de pressão arterial elevada em adolescentes. Arq Bras Cardiol. 2011;96(2):126-33.

29. Christofaro DGD, Ritti-Dias RM, Fernandes R, Polito MD, Andrade $\mathrm{SM}$, Cardoso JR, et al. Detecção de hipertensão arterial em adolescentes através de marcadores gerais e adiposidade abdominal. Arq Bras Cardiol. 2011;96(6):465-70.
30. Fernandéz JR, Redden DT, Pietrobelli A, Allison DB. Waist circumference percentiles in nationally representative samples of African-American, European-American, and Mexican-American children and adolescents. J Pediatr. 2004;145(4):439-44.

31. International Diabetes Federation. The IDF consensus worldwide definition of the metabolic syndrome. International Diabetes Federation; 2005.

32. Rosa ML, Mesquita ET, da Rocha ER, Fonseca Vde M. Índice de massa corporal e circunferência da cintura como marcadores de hipertensão arterial em adolescentes. Arq Bras Cardiol. 2007;88(5):573-8.

33. Taylor RW, Grant AM, Williams SM, Goulding A. Sex differences in regional body fat distribution from pre- to postpuberty. Obesity. 2010;18(7):1410-6.

34. Lazzer S, BoirieY, Meyer M, Vermorei M. Evaluation of foot-to-foot biolectrical impedance analysers to assess body composition in overweight and obese adolescents. Br J Nutr. 2003;90:987-92.

35. Wu YT, Nielsen DH, Cassady SL, Cook JS, Janz KF, Hansen JR. Cross-validation of bioelectrical impedance analysis of body composition in children and adolescents. Phys Ther. 1993;73(5):320-8.

36. Leal GVS, Philippi ST, Matsudo SMM, Toassa EC. Food intake and meal patterns of adolescents, São Paulo, Brazil. Rev Bras Epidemiol. 2010;13(3):457-67.

37. Múnera $N E$, Uscátegui RM, Parra $B E$, Manjarrés LM, Patiño $F$, Velásquez CM, et al. Environmental risk factors and metabolic syndrome components in overweight youngsters. Biomedica. 2012;32:77-91. 